\title{
Mélanocytes épidermiques et mélanocytes folliculaires
}

\author{
J.P. ORTONNE (1) et J.P. BENEDETTO \\ F.R.A. I:N.S.E.R.M. No 11 \\ «Recherche Dermatologique et Immunologie» \\ Clinique Dermatologique (Professeur J. THIVOLET) \\ Hôpital Ed.-Herriot, 69374 Lyon Cedex 2, France
}

\begin{abstract}
Résumé
La couleur de la peau (épiderme, poils, cheveux) des Mammifères est due à la présence de mélanines (eumélanines, phaeomélanines, trichochromes). Ces pigments sont portés par des organes intracellulaires appelés mélanosomes. Ceux-ci sont produits par une cellule spécialisée, le mélanocyte, qui les transfère ultérieurement aux kératinocytes avoisinants par l'intermédiaire de ses dendrites. Chez les Mammifères, l'ensemble de la population mélanocytaire de la peau peut être considéré, dans les conditions normales, comme un système bicompartimental (compartiment épidermique et compartiment folliculaire). L'individualisation de ces deux sous-populations repose sur des bases anatomiques et physiologiques. En effet, si le fonctionnement des mélanocytes est dépendant du programme génétique qui est commun aux cellules pigmentaires des deux compartiments, il peut être influencé par l'environnement interne et externe qui est différent dans l'épiderme et dans le follicule pileux. Cette distinction est importante pour l'étude comparée de la pigmentation cutanée de l'Homme (peau dépourvue de pelage) et des autres Mammifères. Dans les conditions normales, les facteurs les plus importants dans le déterminisme de la pigmentation constitutive de la peau sont : la nature biochimique des mélanines synthétisées et le niveau d'activité des mélanocytes.
\end{abstract}

Chez les Mammifères, les mélanines (eumélanines, phaeomélanines et trichochromes) sont les pigments responsables de la couleur de la peau, des poils et des cheveux (Prota \& Thomson, 1976). Ces pigments sont portés par des organelles intracellulaires appelés mélanosomes. Ceux-ci sont produits par une cellule spécialisée, le mélanocyte, qui les transfère ultérieurement aux kératinocytes avoisinants par l'intermédiaire de ses dendrites (FitzPatrick et al., 1979). Ces mélanocytes se localisent dans les follicules pileux et dans l'assise basale de l'épiderme. Des mélanocytes dermiques ont également été décrits dans certaines espèces et notamment chez l'Homme dans des circonstances très particulières (tache mongolique, naevus de OTA). Quelle que soit leur localisation dans la peau, les mélanocytes ont une origine embryologique commune, la crête neurale. Dans les conditions normales, la population mélanocytaire de la peau des Mammifères peut être considérée comme un système bicompartimental (compartiment épidermique et compartiment folliculaire). Cette distinction n'est pas sans importance lorsque l'on envisage l'étude de la pigmentation cutanée dans la perspective

(1) Nouvelle adresse : Service de dermatologie, Hôpital Pasteur, 06000 Nice. 
d'une comparaison entre l'Homme et les autres Mammifères. En effet, l'Homme se distingue par sa peau nue, dépourvue de pelage. Cette différence morphologique retentit sur la physiologie du système pigmentaire, d'autant plus que le fonctionnement des mélanocytes dépend du programme génétique, mais peut également être modulé par des facteurs internes (facteurs tissulaires, hormones) et externes (ultraviolets...).

Si les deux populations mélanocytaires peuvent être distinguées par leur localisation anatomique et par certains aspects de leur physiologie, elles présentent cependant une certaine unicité. Ainsi, les deux compartiments mélanocytaires ne constituent pas des systèmes clos, puisque des mouvements de mélanocytes entre les follicules pileux et l'épiderme ont pu être mis en évidence.

\section{Origine embryonaire des mélanocytes cutanés}

Les mélanocytes cutanés dérivent de la crête neurale (MinTz, 1967). Leurs précurseurs, les mélanoblastes, correspondent à de grandes cellules rondes ou ovalaires. La différenciation des mélanoblastes en mélanocytes (acquisition du caractère dendritique, positivité de la DOPA réaction) se produit chez l'Homme entre la $8^{\mathrm{e}}$ et la $14^{\mathrm{C}}$ semaine de la vie intra-utérine. Après une migration qui s'effectue selon un axe dorsoventral et craniocaudal, les mélanoblastes atteignent leur territoire définitif, c'est-à-dire l'assise basale de l'épiderme et les follicules pileux. Il est donc clairement établi que les mélanocytes tégumentaires des Mammifères ont pour origine plusieurs clones mélanoblastiques, qui se sont répartis symétriquement à la surface du corps.

Les mélanocytes colonisent donc l'épiderme avant la différenciation des poils. Au stade initial de l'apparition des poils, les mélanocytes se répartissent au hasard sans localisation privilégiée dans l'ébauche pilaire. Ce n'est qu'après le sixième mois de la vie intra-utérine, que les mélanocytes se localiseront à l'infundibulum (assise périphérique de la gaine épithéliale externe) et au sommet de la papule dermique dans le bulbe pileux (Mishima \& Widlan, 1966).

Diverses anomalies du développement embryonnaire peuvent perturber l'installation du système pigmentaire de la peau :

- par atteinte de la crête neurale avec absence de développement des mélanoblastes ou réduction de leur nombre;

- par atteinte du mélanoblaste qui perd sa capacité de différenciation mélanocytaire ;

- par l'influence néfaste de l'environnement tissulaire qui perturbe la migration du mélanoblaste et/ou empêche sa survie.

De telles mutations sont observées chez l'Homme (piébaldisme, syndrome de Waardenburg...) aussi bien que chez la plupart des autres Mammifères. Elles se caractérisent dans l'épiderme et dans les follicules pileux par une absence de mélanocytes (Mosher et al., 1979). 


\section{Les compartiments mélanocytaires épidermiques et folliculaires chez les Mammifères}

Trois grands groupes peuvent être individualisés :

- des Mammifères où le compartiment épidermique est pratiquement inexistant, le compartiment folliculaire étant le plus important. La Souris et le Cobaye représentent l'exemple type de ce groupe. Les mélanocytes sont rares ou absents dans l'épiderme des zones pileuses. Ils sont plus nombreux sur les zones où les poils sont rares ou absents (oreilles, sole plantaire, queue) (Quevedo et al., 1966 ; Billingham \& MEDAWAR, 1953) ;

- des Mammifères où les deux compartiments épidermique et folliculaire sont quantitativement importants. Tel est le cas des grands Singes, en particulier du Chimpanzé où la densité de la population mélanocytaire épidermique est comparable dans les zones pileuses et non pileuses (Post et al., 1966) ;

- chez l'Homme, chez lequel le compartiment épidermique est le plus important. Le compartiment folliculaire n'est cependant pas négligeable. Ainsi, la densité des follicules pileux chez l'Homme est égale ou parfois supérieure à celle des grands Singes. La différence fondamentale réside dans le type de la couverture pileuse. Chez le Singe, cette dernière préserve presque totalement les mélanocytes épidermiques de l'influence des facteurs externes. Au contraire, chez l'Homme, une telle protection est inexistante (SzABo, 1959).

Le mode d'installation du système pigmentaire de la peau pendant la vie embryonnaire suggère qu'une relation très simple pourrait unir les compartiments mélanocytaires de l'épiderme et des follicules pileux : à savoir plus il y a de mélanocytes folliculaires, moins il y a de mélanocytes épidermiques et vice versa. Cependant, les études quantitatives des deux sous-populations de mélanocytes ne vérifient pas cette hypothèse chez la plupart des Mammifères. De plus, chez l'Homme, le nombre des mélanocytes épidermiques dans une zone très pileuse (cuir chevelu) est beaucoup plus élevé que dans des zones à faible pilosité (abdomen).

L'indépendance relative des mélanocytes épidermiques et folliculaires est bien objectivée chez l'Homme au cours de situations physiologiques ou pathologiques. Ainsi, les cheveux gris séniles peuvent être observés sur un cuir chevelu normalement pigmenté. A l'inverse, la pigmentation des poils follets peut persister bien que l'épiderme environnant soit dépigmenté (Mosher et al., 1979).

\section{Caractères communs aux mélanocytes épidermiques et folliculaires}

Le mélanocyte est une cellule dendritique possédant une activité dopa-oxydasique. En microscopie électronique, il se caractérise par un cytoplasme clair, ne contenant ni tonofilaments, ni desmosomes, mais contenant de nombreux microfilaments et des organelles spécifiques, les mélanosomes.

Dans l'épiderme, comme dans les follicules pileux, le mélanocyte entretient des rapports avec les kératinocytes avoisinants auxquels il transfère les granules pigmen- 
taires appelés mélanosomes. Ces kératinocytes vont ultérieurement transporter le pigment et éventuellement le dégrader. L'ensemble du mélanocyte et des kératinocytes qui lui sont associés constitue une unité fonctionnelle, appelée unité épidermique de mélanisation (U.E.M.). A l'intérieur des U.E.M. épidermiques et folliculaires, les diverses étapes de la mélanogénèse se déroulent schématiquement en plusieurs phases successives : la synthèse des mélanosomes, leur mélanisation, leur transfert aux kératinocytes, leur dégradation puis l'élimination de la mélanine.

Le mélanosome est un organelle complexe composé de «membranes », de protéines de structure, de tyrosinase et d'autres enzymes accessoires. Les mélanosomes associés à la synthèse d'eumélanines sont ovales avec une structure filamenteuse. Au contraire, les mélanosomes associés à la synthèse de phaeomélanines sont ronds et granuleux (JimBow \& TAKEUCHI, 1979). La maturation des mélanosomes se traduit par le dépôt progressif de mélanine dans l'organelle. Après avoir migré de la région périnucléaire vers l'extrémité des dendrites, les mélanosomes sont transférés aux kératinocytes avoisinants par divers mécanismes (cytophagocytose, injection directe, ingestion). Dans les kératinocytes, les mélanosomes se répartissent selon leur taille : isolément pour les plus gros, en paquets pour les plus petits. Ils sont secondairement dégradés dans des vacuoles lysosomiales (FitzPatrick et al., 1979).

\section{Caractères distinctifs des U.E.M. épidermiques et folliculaires}

Dans l'épiderme, les U.E.M. se répartissent régulièrement dans l'assise basale. Au contraire, dans les follicules pileux, cette distribution est irrégulière, puisque les mélanocytes actifs se localisent exclusivement dans l'infundibulum et dans le bulbe pileux, au sommet de la papille dermique. Des mélanocytes amélaniques ont également été décrits, dans la partie moyenne des follicules pileux, au niveau de la gaine épithéliale externe (STARICco, 1963). Dans l'épiderme, chaque U.E.M. est formée d'un mélanocyte et de 36 kératinocytes. Ce rapport est beaucoup plus faible dans le follicule pileux.

Bien que les mélanocytes épidermiques et folliculaires dérivent de précurseurs communs et possèdent les mêmes gènes spécifiques de pigmentation, ils se distinguent par une physiologie différente. Ceci résulte probablement d'une modulation (activation ou répression) de l'action de ces gènes de pigmentation par l'environnement tissulaire où le mélanophage a migré.

Les mélanocytes folliculaires synthétisent des mélanosomes qui en général sont plus gros que ceux des mélanocytes épidermiques (Toda et al., 1972). Or il est clairement établi que le mode de répartition des mélanosomes dans les kératinocytes est conditionné par leur taille (KonRAD \& WOLFF, 1973). Les mélanosomes de grande taille se répartissent isolément tandis que les mélanosomes de petite taille se groupent en paquets. Dans les follicules pileux, quelle que soit la race considérée, les mélanosumes se répartissent isolément. Dans l'épiderme, les mélanosomes sont isolés ou groupés, la proportion de l'une ou l'autre de ces catégories variant selon le contexte racial (SzaBo et al., 1972). 


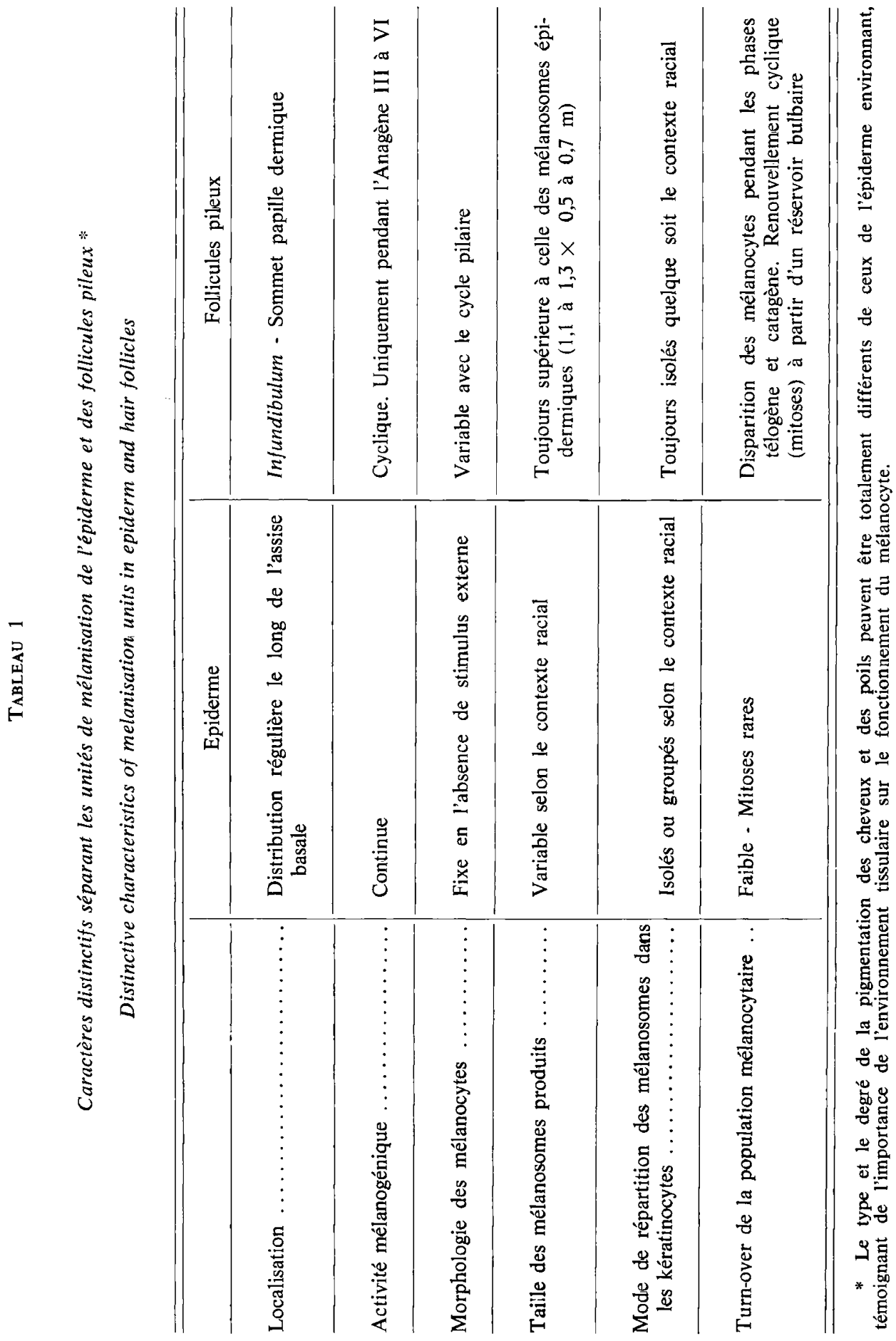


Dans l'épiderme, l'activité des mélanocytes est continue. Elle détermine la pigmentation constitutive de l'individu considéré. L'intervention de stimuli internes ou externes détermine l'acquisition d'une pigmentation facultative. L'activité des mélanocytes folliculaires est au contraire discontinue, rythmée par le cycle pilaire. Ceux-ci ne synthétisent activement du pigment que pendant une phase très courte du cycle pilaire, à savoir de l'anagène III à l'anagène VI. Ces phases de synthèse des mélanocytes se traduisent par des modifications morphologiques (augmentation du nombre des dendrites et du volume du cytoplasme, développement de l'appareil de Golgi et du réticulum endoplasmique, augmentation du nombre de mélanosomes) et biochimiques (apparition d'une activité tyrosinasique dont les formes moléculaires séparables par électrophorèse sur gel de polyacrylamide se modifient au cours du cycle) (BURNETT et al., 1969). L'homéostasie de la population mélanocytaire est différente dans l'épiderme et dans les follicules pileux. Au cours de la vie, la population mélanocytaire de l'épiderme est relativement constante, bien que décroissant progressivement avec l'âge. En l'absence de toute stimulation externe, il est exceptionnel d'observer des images de mitoses mélanocytaires dans l'épiderme. Cependant, plusieurs arguments expérimentaux montrent que la population mélanocytaire de l'épiderme constitue un système dynamique dont le taux de renouvellement est faible mais continu. Par contre, la stimulation par les ultraviolets augmente beaucoup le nombre de mitoses mélanocytaires (ROSDAHL, 1979).

Dans les follicules pileux, la population des mélanocytes bulbaires est renouvelée à chaque cycle. Pendant les phases catagène et télogène, certains mélanocytes bulbaires survivent et se dédifférencient. Ultérieurement, ils prolifèrent, se différencient et repeuplent le bulbe durant le début du stade catagène. L'existence d'un réservoir de mélanocytes dans les follicules pileux des Mammifères est donc très probable (SugiYama \& Kukita, 1976).

Il semble que les réponses des mélanocytes épidermiques et folliculaires à des stimulations hormonales diffèrent. Ainsi, le fonctionnement des mélanocytes folliculaires est profondément modifié par l'ovariectomie, l'œstrogénothérapie et la grossesse. Au contraire, les mélanocytes épidermiques sont comparativement peu affectés. Cette différence de réactivité peut suggérer que le seuil de sensibilité à ces stimulations hormonales est plus élevé pour les mélanocytes épidermiques que pour les mélanocytes folliculaires (SNELL, 1972).

Enfin, il faut souligner que l'influence des facteurs externes s'exerce beaucoup plus sur les mélanocytes épidermiques superficiels que sur les mélanocytes folliculaires situés plus profondément dans le derme. Ceci est en particulier très frappant pour les ultraviolets. Les U.V.B. $(\lambda=290$ à $320 \mathrm{~nm})$ atteignent la basale épidermique et stimulent l'activité mélanogénique des mélanocytes. Au contraire, seule une quantité relativement faible d'U.V.A. $(\lambda=320$ à $400 \mathrm{~nm})$ atteint la partie profonde des follicules pileux, ce qui, dans les conditions normales est insuffisant pour entraîner une stimulation des mélanocytes.

\section{Mouvements cellulaires entre les compartiments mélanocytaires de l'épiderme et des follicules pileux}

La réalité de tels mouvements a été clairement établie. Les deux compartiments mélanocytaires ne constituent donc pas un système clos. 


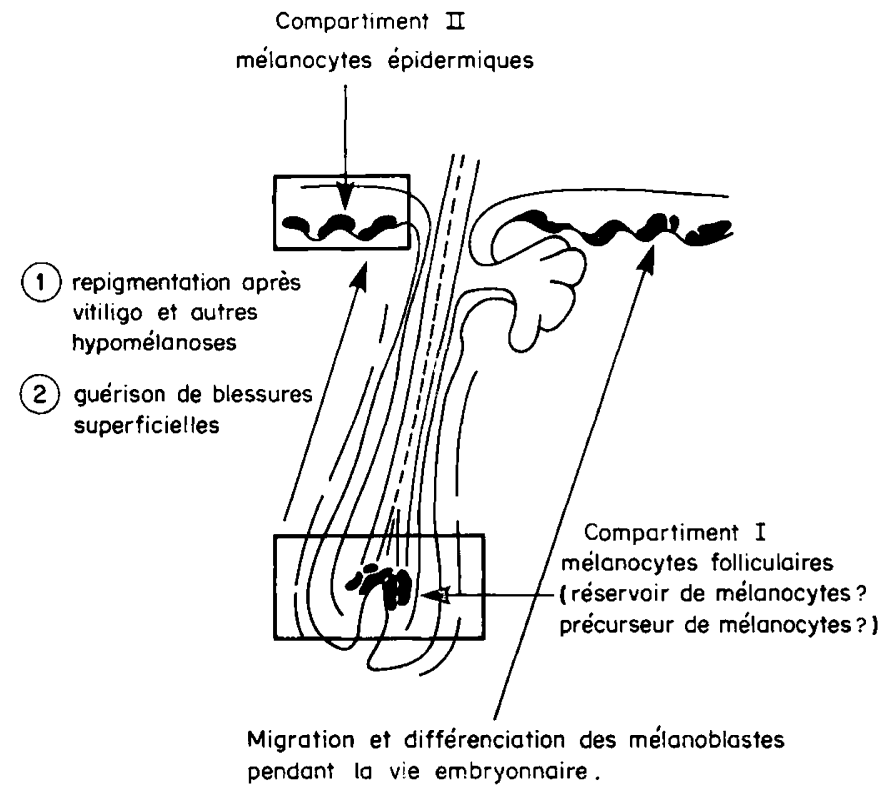

Fic. I

Modèle à 2 compartiments de la population mélanocytaire de la peau A biocompartmental model of the melanocyte population in the skin

Ces mouvements de mélanocytes ont été clairement démontrés dans le sens follicule pileux épiderme. Ils sont observés exclusivement lorsque la population de mélanocytes épidermiques a été détruite, par exemple après une blessure superficielle emportant l'épiderme et l'infundibulum pilaire, mais laissant en place le bulbe pileux. La repigmentation du vitiligo chez l'Homme implique également une migration des mélanocytes du follicule pileux vers l'épiderme. STARIcco (1963) a suggéré que ce sont les mélanocytes amélaniques de la partie moyenne du follicule pileux qui se réactivent, se multiplient, puis migrent vers l'épiderme pour le recoloniser, lors de la cicatrisation d'une blessure superficielle. L'étude de la repigmentation du vitiligo suggère un schéma un peu différent. Il existe dans un premier stade une prolifération de mélanocytes hypertrophiques à la partie profonde du follicule pileux. Ces mélanocytes migrent ensuite le long du follicule jusqu'à l'infundibulum, puis recolonisent l'épiderme par extension centrifuge (ORTONNE et al., 1979; sous presse). Le dernier modèle montre que chez l'Homme, il existe un réservoir folliculaire de mélanocytes qui peut éventuellement reconstituer le compartiment épidermique lorsque ce dernier est détruit. Il sera intéressant de savoir si ces échanges cellulaires se produisent à l'état normal pour maintenir un certain équilibre entre les deux compartiments mélanocytaires de la peau. 
Le passage de mélanocytes de l'épiderme vers les follicules pileux est beaucoup plus hypothétique. Pendant la vie embryonnaire, ce sont les mélanocytes épidermiques qui sont à l'origine de la pigmentation des poils. Ce phénomène n'implique pas cependant un mouvement des mélanocytes car ceux-ci sont incorporés passivement aux ébauches pilaires qui se différencient à partir de l'épiderme. Chez le Mammifère adulte, les mélanocytes épidermiques ne semblent pas capables de coloniser les follicules pileux. Si les mélanocytes folliculaires sont détruits par divers agents traumatiques (froid, rayons $\mathrm{X}$...), les bulbes régénérés restent dépigmentés et produisent des poils blancs (Chase \& RAUCH, 1950 ; TAYLOR, 1949). Cependant, la mélanisation de poils adultes a été observée dans deux circonstances expérimentales particulières :

- chez le Cobaye, une autogreffe de peau noire sur un territoire blanc est laissée en place pendant 7 jours. Elle est ensuite décollée et sur la zone cicatricielle apparaît une pigmentation associée à la présence de mélanocytes actifs. Dans les mois suivants, cette zone se garnit de poils blancs mais dans un délai de 6 à 8 mois, apparaissent des poils noirs qui bientôt garnissent le centre de la zone pigmentée (VouLot, 1966) ;

- chez l'Homme, après une résection dermique pure éliminant la partie moyenne et profonde des follicules pileux, les poils néoformés acquièrent de novo une population mélanocytaire. Il est possible que ces mélanocytes aient une origine épidermique (INABA et al., 1979).

Cependant, aucune de ces expériences n'apporte d'arguments décisifs.

Enfin, une dernière expérience prouve que les mélanocytes épidermiques et folliculaires sont interchangeables. Lorsqu'une suspension de cellules d'un épiderme pigmenté de Cobaye est injectée dans une blessure ayant emporté l'épiderme et la partie des follicules pileux d'une zone blanche de la peau du Cobaye, les mélanocytes "greffés» sont incorporés dans les follicules, ce qui détermine la pousse de poils pigmentés (PEPPER, 1956).

\section{Conclusion}

Chez l'Homme, comme chez les autres Mammifères, la couleur de la peau et des poils est principalement déterminée par le nombre, la taille, le type et le mode de répartition des mélanosomes. Il est particulièrement intéressant de noter que dans les conditions normales, les différences raciales de pigmentation de la peau chez l'Homme ne reposent pas sur des différences numériques de la population mélanocytaire épidermique. Pour une zone déterminée, le nombre de mélanocytes épidermiques est sensiblement identique chez le noir, le blanc ou l'asiatique. Les facteurs prépondérants dans le déterminisme de la couleur de la peau sont donc le type de pigment synthétisé et le niveau d'activité des mélanocytes. Cette constatation s'applique aux autres Mammifères. 


\author{
Summary \\ Epidermic melanocytes and follicular melanocytes
}

Skin color (epidermis, hair) in mammals is determined by melanins (eumelanins giving dark colors and phaeomelanins giving light colors). These pigments are synthetized on intracellular organelles called melanosomes. These melanosomes, produced by a highly specialized cell, the melanocyte, are transfered to the surrounding keratinocytes via the dendrites.

In mammals, the whole melanocyte population of the skin can be regarded as a bicompartmental system (epidermal and follicular compartments). These two subpopulations of melanocytes are anatomically and, at least, to some extent, physiologically distinct. Although, the level of activity of melanocytes depends on pigmentary genes, common to both subpopulations, it may be influenced by environmental factors which are different in the epidermis and in hair bulbs. This concept is important for a comparative study of skin pigmentation in man (hairless skin) and other mammals. In mammals, the biochemical nature of the pigment and the level of activity of melanocytes are the main factors involved in the determinism of constitutive skin color.

\title{
Références bibliographiques
}

Billingham R.E., Medawar P.B., 1953. A study of the branched cells of mammalian epidermis with special reference to the fate of their division products. Phil. Trans. Soc. B., 237, 151-171.

Burnett J.B., Holstein T.J., Quevedo W.C. Jr., 1979. Electrophoretic variations of tyrosinase in follicular melanocytes during the hair growth cycle in Mice. J. Expl. Zool., 171, 369-376.

Chase H.B., Rauch H., 1950. Greying of hair. II. - Response of individual hairs in Mice to variations in X-irradiation. J. Morph., 87, 381-392.

FitzPatrick T.B, Szabo G., Seiji M., Quevedo W.C. Jr., 1979. Biology of the melanin pigmentary system. In : Dermatology in general medicine, T.B. FitzPaTRICK, A.Z. EISEN, K. Wolff, I.M. Freedberg, K.F. Austen, Mc Graw Hill Book Company, 131-163.

Inaba M., Anthony J., Mc Kinstry C., 1979. Histologic study of the regeneration of axillary hair after removal with subcutaneous tissue shaver. J. Invest. Derm., 72, 224-231.

Jimbow K., TAKeUChi T., 1979. Ultrastructural comparison of phaeo and eumelanogenesis in animals. Pigment Cell, 4, Karger Basel, 308-317.

KonRaD K., WolfF K., 1973. Hyperpigmentation, melanosome size and distribution patterns of melanosomes. Arch. Dermat., 107, 858-860.

Mintz B., 1967. Gene control of mammalian pigmentary differentiation. I. - Clonal origin of melanocytes. Proc. Nat. Acad. Sci., U.S.A., 58, 344-351.

Mishima Y., Widlan S., 1966. Embryonic development of melanocytes in human hair and epidermis. Their cellular differentiation and melanogenic activity. J. Invest. Derm., 46, 263-277.

Mosher D.B., FitzPatrick T.B., Ortonne J.P., 1979. Abnormalities of pigmentation. In : Dermatology in general medicine, FitzPatrick T.B., Eisen A.Z., WolfF K., FreedBERG I.M., AUSTEN K.F., Mc Graw-Hill Book Company, 568-629.

Ortonne J.P., MaC Donald D.M., Micoud A., Thivolet J., 1979. PUVA induced repigmentation of vitiligo : histoenzymological (split dopa) and ultrastructural study. Brit. J. Derm., 101, 1-13.

Ortonne J.P., Schmitt D., Thivolet J., 1980. PUVA induced repigmentation of vitiligo. Scanning electron microscopy of hair follicules. J. Invest. Derm., 74, 40-42. 
PePper F.J., 1956. Pigmentation in a white haired region of the hooded rat as a result of cell transplantation. J. Morph., 98, 367-387.

Post P.W., Szabo G., Keeling M.E., 1975. A quantitative and morphological study of the pigmentary system of the chimpanzee with the light and electron microscope. Am. $J$. Phys. Anthropology, 43, 435-444.

Prota G., Thomson R.H., 1976. Melanin pigmentation in mammals. Ebdeavour, 35, 32-38.

Quevedo W.C. Ir., Youle M.C., Rovee D.T., Bienecki T.C., 1966. The developmental fate of melanocyte in murine skin. In : Structure control of the melanocytes. G. Della Porta, O. Muhlock eds, Springer Verlag, New York, 228-241.

Rosdahl I., 1979. The epidermal melanocyte population and its reaction to ultraviolet light. Acta Derm. Vener., supp. 88, 1-43.

SNELL R.S., 1972. Hormonal control of hair color. In : Pigmentation : its genesis and biologic control. V. Riley ed., Appleton Century Crofts, New York, 193-205.

STARICco R.G., 1963. Amelanotic melanocytes in the outer sheat of the human hair follicle and their role in the repigmentation of regenerated epidermis. Ann. N.Y. Acad. Sci., 100, 239-255.

Sugyiama S., Kukita A., 1976. Melanocyte reservoir in the hair follicles during the hair growth cycle : an electron microscopic study. In : Biology and diseases of the hair (T. Kobori, W. Montagna eds), University Park Press, Baltimore, 181-200.

SzABO G., 1959. Quantitative histological investigations on the melanocytes system of the human epidermis. In : Pigment cell biology, Gordon M. ed, Academic Press, New York, 99-125.

Szabo G., Gerald A.B., Pathak M.A., Fitzpatrick T.B., 1972. The ultrastructure of racial color differences in Man. In : Pigmentation : its genesis and biologic control. V. Riley, ed, Appleton Century Crofts, New York, 23-41.

TAYLOR A.C., 1949. Survival of rat skin and changes in hair pigmentation following freezing. J. Exp. Zool., 110, 77-112.

Toda K., Pathak M.A., Parrish J.A., Fitzpatrick T.B., 1972. Alteration of racial differences in melanosome distribution in human epidermis after exposure to ultraviolet light. Nature (New Biol.), 236, 143-145.

Voulot C., 1966. Mise en évidence d'une mélanisation des bulbes pileux au cours du phénomène d'extension pigmentaire. C.R. Acad. Sci. Paris, 262, 2646-2649. 